Claremont Colleges

Scholarship@ Claremont

All HMC Faculty Publications and Research

HMC Faculty Scholarship

8-1-1996

\title{
Positive Solutions for a Semilinear Elliptic Problem with Critical Exponent
}

Ismail Ali

Kuwait University

Alfonso Castro

Harvey Mudd College

\section{Recommended Citation}

Ali, Ismail and Alfonso Castro. "Positive solutions for a semilinear elliptic problem with critical exponent", Nonlinear Analysis T.M.A., Vol. 27, No. 3 (1996), pp. 327-338.

This Article - postprint is brought to you for free and open access by the HMC Faculty Scholarship at Scholarship @ Claremont. It has been accepted for inclusion in All HMC Faculty Publications and Research by an authorized administrator of Scholarship @ Claremont. For more information, please contact scholarship@cuc.claremont.edu. 


\title{
0362-546X(95)00059-3
}

\section{POSITIVE SOLUTIONS FOR A SEMILINEAR ELLIPTIC PROBLEM WITH CRITICAL EXPONENT}

\author{
ISMAIL ALI $\uparrow \S$ and ALFONSO CASTRO $\ddagger \|$ \\ † Department of Mathematics, Kuwait University, P.O. Box 5969, Kuwait; and \\ $\ddagger$ Department of Mathematics, University of North Texas, Denton, TX 76203-5116, U.S.A.
}

(Received 1 June 1994; received for publication 21 March 1995)

Key words and phrases: Elliptic boundary value problem, critical exponent, radial solution.

\section{INTRODUCTION}

The solvability of boundary value problems of the form

$$
\Delta u+f(u)=g(x) \text { in } \Omega, \quad u(x)=0 \text { on } \partial \Omega,
$$

where $\Omega$ is a smooth bounded region in $R^{N}, N \geq 3$, and $\Delta$ is the Laplacian operator, depends on the growth of the nonlinearity $f$. We say that $f$ grows subcritically if there exists

$$
q \in(1,(N+2) /(N-2))
$$

such that $\lim \sup _{|u| \rightarrow \infty}|f(u)| /|u|^{q}<\infty$. If $\lim _{|u| \rightarrow \infty}\left(|f(u)| /|u|^{(N+2) /(N-2)}\right) \in R$ then we say that $f$ grows critically. In order to apply to this problem compactness techniques such as those derived from the imbedding properties of the Sobolev spaces (see [1]) one realizes that $f$ must grow subcritically. Moreover, in [2], Pohozaev showed results for the subcritical case that do not extend to the critical case. Here we show, in particular, the existence of large positive solutions for small values of $g$ when $f$ grows critically, which is not the case when $f$ grows subcritically. For related problems with critical exponents the reader is referred to [3-8].

From now on we consider the boundary value problem (1.1) when $\Omega$ is the unit ball in $R^{N}$, $f(u)=|u|^{p} u$ with $p=4 /(N-2)$, and $g(x) \equiv-\lambda \in R$. Our main result is the following theorem.

THEOREM 1. There exists a continuous function $F:(0, \infty) \rightarrow(0, \infty)$ such that $u$ is a positive solution to (1.1) if and only if $\lambda=F(u(0))$. If $u_{1}$ and $u_{2}$ are positive solutions to (1.1) with $u_{1}(0)=u_{2}(0)$ then $u_{1} \equiv u_{2}$. Moreover, $\lim _{d \rightarrow 0} F(d)=0, \lim _{d \rightarrow \infty} F(d)=0$, and there exists $\lambda_{0}>0$ such that if $0<\lambda<\lambda_{0}$ then (1.1) has exactly two solutions. In particular, $\{(\lambda, u) ; u>0$, $u$ satisfies (1.1) $]$ is connected.

The classical work of Gidas et al. [7] tells us that positive solutions in $\Omega$ are radially symmetric. This allows us to shift our study to the ordinary differential equation

§ Partially supported by Kuwait University grant No. SM089.

\| Partially supported by NSF grant DMS 9215027. 


$$
\begin{gathered}
u^{\prime \prime}+\frac{N-1}{r} u^{\prime}+|u|^{p} u+\lambda=0, \quad r \in(0,1), \\
u^{\prime}(0)=0, \\
u(1)=0 .
\end{gathered}
$$

Instead of considering directly this boundary value problem we study $u(r, \lambda, d)$, the solution to the initial value problem (1.2), (1.3), and

$$
u(0)=d .
$$

In order to prove theorem 1 we establish that $u_{\lambda}(1, \lambda, d)<0$ and that for $d>0$ large, $u_{d}(1, \lambda, d)<0$ when $u(\cdot, \lambda, d)$ satisfies (1.2), (1.3), (1.4).

\section{PRELIMINARIES}

First we establish "Pohazaev identity" (see $[2,9])$ for the initial value problem (1.2), (1.3), (1.5). Given $d \in R$, and $\lambda \in R$, define

$$
E(r, \lambda, d)=\frac{\left(u^{\prime}(r, \lambda, d)\right)^{2}}{2}+\frac{(u(r, \lambda, d))^{p+2}}{p+2}+\lambda u(r, \lambda, d) .
$$

Lemma 2. Let $u(r, \lambda, d)$ be a solution to (1.2), (1.3) and (1.5). If $0 \leq \bar{r} \leq r$, then

$$
r^{N-1} H(r)-(\vec{r})^{N-1} H(\vec{r})=\frac{N+2}{2} \int_{\bar{r}}^{r} \lambda s^{N-1} u(s) \mathrm{d} s,
$$

where

$$
H(r)=r E(r)+\frac{N-2}{2} u(r, \lambda, d) u^{\prime}(r, \lambda, d)
$$

Proof. Multiplying (1.2) by $r^{N} u^{\prime}(r)$ and integrating over $[\bar{r}, r]$, we obtain,

$$
r^{N} E(r)=\int_{\vec{r}}^{r} s^{N-1}\left\{N\left(\frac{u^{p+2}(s)}{p+2}+\lambda u\right)-\left(\frac{N-2}{2}\right)\left(u^{\prime}(s)\right)^{2}\right\} \mathrm{d} s+(\bar{r})^{N} E(\bar{r}) .
$$

Similarly, multiplying (1.2) by $r^{N-1} u(r)$ and integrating over $[\bar{r}, r]$ we infer,

$$
\int_{\bar{r}}^{r} s^{N-1}\left(u^{\prime}(s)\right)^{2} \mathrm{~d} s=u^{\prime}(r) u(r) r^{N-1}-u^{\prime}(\bar{r}) u(\bar{r})(\bar{r})^{N-1}+\int_{\bar{r}}^{r} s^{N-1}\left(u^{p+2}(s)+\lambda u\right) \mathrm{d} s .
$$

Replacing (2.4) in (2.3), we obtain (2.2). This completes the proof.

Taking $\bar{r}=0$ in (2.2) we get

$$
\frac{N+2}{2} \int_{0}^{r} \lambda s^{N-1} u(s) \mathrm{d} s=\frac{r^{N}\left(u^{\prime}(r)\right)^{2}}{2}+\frac{r^{N}\left(u^{p+2}(r)\right)}{p+2}+r^{N} \lambda u(r)+\frac{N-2}{2} r^{N-1} u^{\prime}(r) u(r) \text {. }
$$

CoROllary 3. The problem (1.1) has no nonnegative solutions for $\lambda \leq 0$. 
Proof. Taking $\bar{r}=0, r=1$ in (2.2) we obtain

$$
\frac{\left(u^{\prime}(1)\right)^{2}}{2}=\frac{N+2}{2} \int_{0}^{r} \lambda s^{N-1} u(s) \mathrm{d} s .
$$

Since $u$ is positive, (2.6) yields $\left(u^{\prime}(1)\right)^{2} \leq 0$ for $\lambda \leq 0$. Hence, $u \equiv 0$. This completes the proof.

Now, for a positive solution $u$ of (1.1), we define the function

$$
h(r)=-\frac{r u^{\prime}(r)}{u(r)}, \quad r \in[0,1) .
$$

Clearly, $h$ is continuous, and $h(0)=0$. Since $u(1)=0$, we see that $\lim _{r \rightarrow 1^{-}} h(r)=\infty$. Furthermore, $h$ is an increasing function. Indeed,

$$
h^{\prime}(r)=\frac{-u^{\prime}(r) u(r)-r u(r) u^{\prime \prime}(r)+r\left(u^{\prime}(r)\right)^{2}}{(u(r))^{2}} .
$$

Substituting (1.2) in (2.8) we have,

$$
h^{\prime}(r)=\frac{(N-2) u^{\prime}(r) u(r)-r u^{p+2}(r)+r \lambda u(r)+r\left(u^{\prime}(r)\right)^{2}}{(u(r))^{2}} .
$$

Combining (2.5) and (2.9) we have,

$$
h^{\prime}(r)=\frac{\lambda(N+2) r^{1-N} \int_{0}^{r} s^{N-1} u(s) \mathrm{d} s-r \lambda u(r)+(1-2 /(p+2)) r u^{p+2}(r)}{(u(r))^{2}} .
$$

Since $u$ is a decreasing function, it follows from $(2.10)$ that

$$
\begin{aligned}
h^{\prime}(r) & \geq \frac{(1-2 /(p+2)) r u^{p+2}(r)+(2 / N) \lambda r u(r)}{(u(r))^{2}} \\
& \geq\left(1-\frac{2}{p+2}\right) r u^{p}(r)+\frac{2}{N} \lambda r u(r)=\frac{2}{N} r u^{p}(r)>0 .
\end{aligned}
$$

LEMMA 4. If $u$ is a positive solution to $(1.1)$, then there exists $M_{0}>0$ and a unique $\bar{r} \in(0,1)$ such that $\bar{u}(\vec{r})=M_{0} \bar{r}^{-2 / p}$. Moreover, if $0<M<M_{0}$ then there exists exactly two numbers $r_{1}, r_{2} \in(0,1)$ such that $u\left(r_{i}\right)=M\left(r_{i}\right)^{-2 / p}, i=1,2$.

Proof. Let $\bar{r} \in[0,1]$ be such that $M_{0}=\max \left\{u(r) r^{2 / p}: r \in[0,1]\right\}=u(\bar{r}) \bar{r}^{2 / p}$. Thus, the graph of $u$ is tangent to the graph of $M_{0} r^{-2 / p}$, at $\bar{r}$, and $u(r) \leq M_{0} r^{-2 / p}$ for all $r \in[0,1]$.

Now for $M<M_{0}$ we show that the graph of $u$ intersects the graph of $M r^{-2 / p}$ at exactly two points. Suppose $0<r_{1}<r_{2}<r_{3}<1$ are the first three numbers such that $u\left(r_{i}\right)=M r_{i}^{-2 / p}$, $i=1,2,3$. Since $u$ is a decreasing function, $u\left(r_{1}\right)<u\left(r_{2}\right)<u\left(r_{3}\right)$. Let $Z=M r^{-2 / p}$, then, we have $Z\left(r_{2}\right)=u\left(r_{2}\right), Z^{\prime}\left(r_{2}\right)>u^{\prime}\left(r_{2}\right), Z\left(r_{3}\right)=u\left(r_{3}\right)$, and $Z^{\prime}\left(r_{3}\right)<u^{\prime}\left(r_{3}\right)$. Hence,

$$
h\left(r_{3}\right)=\frac{-r_{3} u^{\prime}\left(r_{3}\right)}{u\left(r_{3}\right)}<\frac{-r_{3} Z^{\prime}\left(r_{3}\right)}{Z\left(r_{3}\right)}=\frac{2}{p},
$$


and,

$$
h\left(r_{2}\right)=\frac{-r_{2} u^{\prime}\left(r_{2}\right)}{u\left(r_{2}\right)}>\frac{-r_{2} Z^{\prime}\left(r_{2}\right)}{Z\left(r_{2}\right)}=\frac{2}{p} .
$$

However, then $h\left(r_{3}\right)<h\left(r_{2}\right)$ with $r_{2}<r_{3}$, which contradicts that $h$ is an increasing function (see (2.11)). Assuming that $u(\vec{r}) \bar{r}^{2 / p}=u(\hat{r}) \hat{r}^{2 / p}=M_{0}$ we see that $h(\vec{r})=h(\hat{r})=2 / p$ which contradicts that $h$ is an increasing function. Hence, $\bar{r}$ is unique.

From (2.5) and the quadratic formula we obtain,

$$
r u^{\prime}(r)=-\frac{N-2}{2} u(r) \pm \frac{1}{2} A(r)
$$

where

$$
A(r)=\left\{(N-2)^{2} u^{2}(r)-\frac{8}{p+2} r^{2} u^{p+2}(r)-8 r^{2} \lambda u(r)+4(N+2) \lambda \frac{1}{r^{N-2}} \int_{0}^{r} s^{N-1} u(s) \mathrm{d} s\right\}^{1 / 2}
$$

From (2.12) we have

$$
\frac{2}{N-2} h(r)=1 \pm \frac{1}{N-2} \frac{A(r)}{u(r)} .
$$

Since $h(0)=0$ and $\lim _{r \rightarrow 1^{-}} h(r)=\infty$ we see from (2.14) that for $r$ near zero

$$
\frac{2}{N-2} h(r)=1-\frac{1}{N-2} \frac{A(r)}{u(r)},
$$

and for $r$ near 1 ,

$$
\frac{2}{N-2} h(r)=1+\frac{1}{N-2} \frac{A(r)}{u(r)} .
$$

The fact that $h$ is an increasing function together with (2.15), and (2.16) imply the existence of a unique $\hat{r}$ such that $(2 /(N-2)) h(\hat{r})=1$, that is, $A(\hat{r})=0$. Since $A(\hat{r})=0$ implies $h(\hat{r})=2 / p$ and $\bar{r}$ is the only element in $[0,1]$ for which $h(r)=2 / p$ we see that $\bar{r}=\hat{r}$. Using that $u(\hat{r})=$ $M_{0} \hat{r}^{-2 / p}$ and integrating (2.11) on $[0, \vec{r}]$ we obtain

$$
M_{0} \leq\left(\frac{N(N-2)}{2}\right)^{1 / p}
$$

LeMmA 5. If $\hat{r}$ is as above, then $\hat{r} \leq O\left(d^{-p / 2}\right)$.

Proof. Let $r_{0}=d^{-p / 2}$, and put $K_{0}=r_{0}^{2} u^{p}\left(r_{0}\right)$. We claim that $K_{0} \geq(1-1 / 4 N)^{p}$. Indeed, since

$$
r^{N-1} u^{\prime}(r)=-\int_{0}^{r} s^{N-1}\left(\lambda+u^{p+1}(s)\right) \mathrm{d} s \geq-\frac{d^{p+1}+\lambda}{N} r^{N},
$$

it follows that $u^{\prime}(r) \geq-\left(\left(d^{p+1}+\lambda\right) / N\right) r$. Integration over $\left[0, r_{0}\right]$ yields

$$
u\left(r_{0}\right) \geq u(0)-\frac{d^{p+1}+\lambda}{2 N} r_{0}^{2}=d-\frac{d^{p+1}+\lambda}{2 N} d^{-p}=\frac{2 N-1}{N} d-\frac{\lambda}{2 N} d^{-p} .
$$


Thus, for $\lambda \in[0,1]$ and $d$ large, $K_{0}^{1 / p} r_{0}^{-2 / p}=u\left(r_{0}\right) \geq((2 N-1) / 3 N) d$. Hence, $K_{0}^{1 / p} \geq$ $(2 N-1) / 3 N$ and the claim is established.

If $\hat{r} \geq d^{-p / 2}$, then integrating $(2 /(N-2)) h^{\prime}(r)$ over $\left[r_{0}, \hat{r}\right]$ and using (2.11) we obtain

$$
1=\frac{2}{N-2} h(\hat{r}) \geq \frac{2}{N-2} h\left(r_{0}\right)+\frac{4}{N(N-2)} \int_{r_{0}}^{\hat{r}} K_{0} r^{-2} r \mathrm{~d} r .
$$

Hence

$$
\ln \left(\frac{\hat{r}}{r_{0}}\right) \leq \frac{N(N-2)}{4 K_{0}}, \quad \text { or equivalently, } \hat{r} \leq d^{-p / 2} \exp \left(\frac{N(N-2)}{4 K_{0}}\right),
$$

which proves lemma 5 .

LEMMA 6. If $u$ is a solution to (1.2), (1.3) and (1.5), then

$$
\frac{2}{p} d u_{d}(r)=-\frac{N+2}{2} \lambda u_{\lambda}(r)+\frac{N-2}{2} u(r)+r u^{\prime}(r) .
$$

Proof. Let $v(r)=\beta^{-2 / p} u(r / \beta, \lambda, d)$. Thus,

$$
\begin{aligned}
v^{\prime \prime}+\frac{N-1}{r} v^{\prime}+v^{p+1} & =\frac{1}{\beta^{2+2 / p}} u^{\prime \prime}\left(\frac{r}{\beta}\right)+\frac{1}{\beta^{1+2 / p}} \cdot \frac{N-1}{r} u^{\prime}\left(\frac{r}{\beta}\right)+\frac{1}{\beta^{2+2 / p}} u^{p+1}\left(\frac{r}{\beta}\right) \\
& =\frac{1}{\beta^{2+2 / p}} u^{\prime \prime}\left(\frac{r}{\beta}\right)+\frac{1}{\beta^{2+2 / p}} \cdot \frac{\beta(N-1)}{r} u^{\prime}\left(\frac{r}{\beta}\right)+\frac{1}{\beta^{2+2 / p}} u^{p+1}\left(\frac{r}{\beta}\right) \\
& =\frac{1}{\beta^{2+2 / p}}(-\lambda),
\end{aligned}
$$

which implies that $v(r)=u\left(r, \lambda / \beta^{2+2 / p}, d / \beta^{2 / p}\right)$. Thus, for all $\beta>0$,

$$
u(r / \beta, \lambda, d)=\beta^{2 / p} u\left(r, \lambda / \beta^{2+2 / p}, d / \beta\right) .
$$

Differentiating (2.19) with respect to $\beta$ we obtain, for all $r>0, \lambda>0, d>0, \beta>0$,

$$
\begin{aligned}
-\frac{r}{\beta^{2}} u^{\prime}\left(\frac{r}{\beta}, \lambda, d\right)= & \frac{2}{p} \beta^{2 / p-1} u\left(r, \frac{\lambda}{\beta^{2+2 / p}}, \frac{d}{\beta^{2 / p}}\right)-\left(2+\frac{2}{p}\right) \beta^{-3} u_{\lambda}\left(r, \frac{\lambda}{\beta^{2+2 / p}}, \frac{d}{\beta^{2 / p}}\right) \\
& -\left(\frac{2 d}{p}\right) \beta^{-1} u_{d}\left(r, \frac{\lambda}{\beta^{2+2 / p}}, \frac{d}{\beta^{2 / p}}\right) .
\end{aligned}
$$

In particular, taking $\beta=1$, we obtain (2.18). This completes the proof.

Substituting (2.12) in (2.18) we have

$$
\frac{N-2}{2} d u_{d}(r)=-\frac{N-2}{2} \lambda u_{\lambda}(r) \pm \frac{1}{2} A(r) .
$$

In (2.20) the " + " sign is to be used on the interval $[0, \hat{r}]$, and the " - " sign on the interval $(\hat{r}, 1]$ (see (2.15) and (2.16)). The existence and uniqueness of the number $\hat{r}$ are due to the fact that $h(r)=-r u^{\prime} / u$ is an increasing function. 
LEMMA 7. If $u$ is a positive solution to (1.2), (1.3) and (1.5) then

$$
-\frac{1}{2 N} \leq-\frac{r^{2}}{2 N} \leq u_{\lambda}(r, \lambda, d) \leq 0 \quad \text { for } r \in[0,1] .
$$

Proof. Let $z(r)=u_{\lambda}(r, \lambda, d)$. Thus,

$$
z^{\prime \prime}+\frac{N-1}{r} z^{\prime}+(p+1)|u|^{p} z+1=0, \quad z(0)=0, \quad \text { and } \quad z^{\prime}(0)=0 .
$$

Let $G$ be defined by

$$
G(r)=\frac{1}{2}\left(z^{\prime}\right)^{2}+\frac{1}{2}(p+1)|u|^{p} z^{2}+z .
$$

Differentiating (2.22) we obtain

$$
\begin{aligned}
G^{\prime}(r) & =z^{\prime} z^{\prime \prime}+(p+1)|u|^{p-1} z\left[z^{\prime}|u|+\frac{p}{2} u^{\prime} z\right]+z^{\prime} \\
& =-\frac{(N-1)}{r}\left(z^{\prime}\right)^{2}+\frac{1}{2}(p+1)|u|^{p} u^{\prime} z^{2} \leq 0,
\end{aligned}
$$

where we have used that $u^{\prime} \leq 0$.

Suppose, there is an $\tilde{r}>0$ such that $z(\tilde{r})=0$. Then substituting in (2.22) yields $G(\tilde{r})=$ $\frac{1}{2}\left(z^{\prime}(\tilde{r})\right)^{2} \geq 0$, contradicting the fact that $G$ is a decreasing function. Therefore, $z \leq 0$ on $[0,1]$. However, then

$$
r^{N-1} z^{\prime}(r)=-\int_{0}^{r} s^{N-1}\left(1+(p+1)|u|^{p}(u) z\right) \mathrm{d} s \geq \int_{0}^{r} s^{N-1} \mathrm{~d} s
$$

and, hence, $z^{\prime}(r) \geq-r / N$. Integrating over $[0, r]$ we obtain

$$
z(r) \geq z(0)-\frac{r^{2}}{2 N}=-\frac{r^{2}}{2 N} \geq-\frac{1}{2 N} .
$$

This completes the proof.

From (2.20), lemma 7, and the definition of $\hat{r}$ we obtain that

$$
w(r)=u_{d}(r, \lambda, d)>0, \quad \text { for } r \in[0, \tilde{r}] .
$$

LEMMA 8. If $u\left(r_{2}\right)=((N-2) / 4(N+2))^{1 / p} r_{2}^{-2 / p}$, with $0<\hat{r} \leq r_{2}<1$ then $r_{2} \leq O\left(d^{-p / 2}\right)$.

Proof. Since

$$
\frac{2}{N-2}\left(h\left(r_{2}\right)-h(\hat{r})\right)=\sqrt{\frac{6 N-4}{N(N+2)}+O\left(r^{2+2 / p}\right)},
$$

and $(u(r))^{p} \geq \frac{1}{4}\left((N-2)^{3} /(N+2)\right)\left(1 / r^{2}\right)$, for $r \in\left(\hat{r}, r_{2}\right)$; from (2.11) we have

$$
\sqrt{\frac{6 N-4}{N(N+2)}+O\left(r^{2+2 / p}\right)} \geq \frac{(N-2)^{2}}{N(N+2)} \int_{r}^{r_{2}} r^{-1} \mathrm{~d} r
$$


Therefore,

$$
\ln \left(\frac{r_{2}}{\hat{r}}\right) \leq \frac{\sqrt{N(N+2)(6 N-4)}}{(N-2)^{2}}=\delta(N),
$$

that is, $r_{2} \leq \hat{r} \exp (\delta(N))$. Since from lemma $5, \hat{r}=O\left(d^{-p / 2}\right)$, we conclude that $r_{2}=O\left(d^{-p / 2}\right)$. This completes the proof.

Now we show that for $d$ sufficiently large $u_{d}\left(r_{2}\right)<0$. Indeed,

$$
\left(\frac{2}{p}\right) \mathrm{d} u_{d}\left(r_{2}\right)=-\lambda \frac{N+2}{2} u_{\lambda}\left(r_{2}\right)-\frac{1}{2} A\left(r_{2}\right),
$$

and since $u_{\lambda}(r) \geq-r^{2} / 2 N$ (see lemma 7) we have

$$
\begin{aligned}
\left(\frac{2}{p}\right) \mathrm{d} u_{d}\left(r_{2}\right) \leq & \lambda \frac{N+2}{2 N} r_{2}^{2} \\
& -\sqrt{r_{2}^{-4 / p}\left(\frac{(N-2)^{3}}{4(N+2)}\right)^{2 / p} \frac{6 N-4}{N(N+2)}-\lambda\left(8-\frac{4(N+2)}{N}\right)\left(\frac{(N-2)}{4(N+2))^{1 / p} r_{2}^{2-2 / p}} .\right.}
\end{aligned}
$$

Simplifying the expression on the right we get

$$
\left(\frac{2}{p}\right) \mathrm{d} u_{d}\left(r_{2}\right) \leq \lambda \frac{N+2}{4 N} r_{2}^{2}-\frac{N-2}{2} r_{2}^{-2 / p}\left(\frac{(N-2)^{3}}{4(N+2)}\right)^{1 / p} \sqrt{\frac{6 N-4}{N(N+2)}+O\left(r_{2}^{2-2 / p}\right)} .
$$

From lemma 8 we obtain

$$
\left(\frac{2}{p}\right) \mathrm{d} u_{d}\left(r_{2}\right) \leq \lambda \frac{N+2}{2 N} r_{2}^{2}-O(d)
$$

and, hence,

$$
u_{d}\left(r_{2}\right) \leq-O(1)+O\left(d^{-1}\right)<0, \quad \text { for } d \text { sufficiently large. }
$$

From (2.24) and (2.27) we see that there exists an $\tilde{r} \in\left(\hat{r}, r_{2}\right)$ such that

$$
u_{d}(\tilde{r})=0
$$

Let $\gamma(r)=r^{-(N-2) / 2}$. A straightforward calculation shows that $\gamma^{\prime \prime}+((N-1) / r) \gamma^{\prime}+$ $((N-2) / 2 r)^{2} \gamma=0$. Since $u_{d}$ satisfies a linear differential equation, its zeros are nondegenerate. Since $(p+1) u^{p}(r) \leq\left((N-2)^{2} / 4 r^{2}\right)$ for $r \in\left[r_{2}, 1\right)$, and $\gamma$ is positive on $(0, \infty)$, by the Sturm comparison theorem (see [10]) we see that $u_{d}(\cdot, \lambda, d)$ cannot have two zeros in $\left[r_{2}, 1\right]$. The next three lemmas are devoted to proving that $u_{d}(\cdot, \lambda, d)<0$ in $\left(r_{2}, 1\right]$.

LEMMA 9. Let $u$ be a positive solution to (1.2), (1.3) and (1.5). Then

$$
\lim _{d \rightarrow+\infty} \int_{0}^{1} r^{N-1} u(r, \lambda, d) \mathrm{d} r=0
$$


Proof. Let $\varepsilon>0$ be such that for $r>\hat{r}+\delta,(2 /(N-2)) h(r) \geq 1+\varepsilon$ (see (2.16)). Thus,

$$
-\frac{u^{\prime}}{u} \geq \frac{1}{2}(1+\varepsilon)(N-2) \frac{1}{r} \text {. }
$$

Integrating this over $\left[r_{2}, r\right]$ we get

$$
u(r) \leq U\left(r_{2}\right) r_{2}^{(1 / 2)(1+\varepsilon)(N-2)} r^{-(1 / 2)(1+\varepsilon)(N-2)},
$$

which in turn gives that

$$
u(r) \leq\left(\frac{(N-2)^{3}}{4(N+2)}\right)^{1 / p} r_{2}^{((N-2) / 2) \varepsilon} r^{-((N-2) / 2)(1+\varepsilon)}
$$

Therefore,

$$
\int_{r_{2}}^{1} r^{N-1} u(r) \mathrm{d} r \leq\left(\frac{(N-2)^{3}}{4(N+2)}\right)^{1 / p} r_{2}^{N-\varepsilon((N-2) / 2) \varepsilon} \int_{r_{2}}^{1} r^{(N-\varepsilon(N-2)) / 2} \mathrm{~d} r
$$

and, hence,

$$
\int_{r_{2}}^{1} r^{N-1} u(r) \mathrm{d} r \leq\left(\frac{(N-2)^{3}}{4(N+2)}\right)^{1 / p} \frac{2 r_{2}^{((N-2) / 2) \varepsilon}}{2 N-(1+\varepsilon)(N-2)},
$$

which tends to zero as $d$ tends to $+\infty$, since $r_{2} \rightarrow 0$ as $d \rightarrow \infty$. Also, since

$$
\int_{0}^{r_{2}} r^{N-1} u(r) \mathrm{d} r \leq \frac{d}{N} r_{2}^{N}=\frac{d}{N}\left(d^{-p / 2}\right)^{N}=\frac{1}{N} d^{-(2+N) /(N-2)},
$$

which tends to zero as $d \rightarrow+\infty$. We conclude that

$$
\int_{0}^{1} r^{N-1} u(r) \mathrm{d} r \rightarrow 0 \quad \text { as } d \rightarrow+\infty .
$$

This completes the proof.

LemMa 10. If $u$ is a positive solution to (1.2), (1.3) and (1.5) then

$$
\int_{0}^{1} r^{N-1} u^{p+1} \mathrm{~d} r \leq o(\sqrt{\lambda}) .
$$

Proof. Taking $r=1$ in Pohozaev's identity (2.5) we obtain

$$
\left(u^{\prime}(1)\right)^{2}=\lambda(N+2) \int_{0}^{1} r^{N-1} u(r) \mathrm{d} r
$$

Hence,

$$
\left(\int_{0}^{1} r^{N-1} u^{p+1} \mathrm{~d} r\right)^{2} \leq\left(\int_{0}^{1} r^{N-1}\left(\lambda+u^{p+1}\right) \mathrm{d} r\right)^{2}=\left(u^{\prime}(1)\right)^{2} .
$$

This and lemma 9 yield

$$
\left(\int_{0}^{1} r^{N-1} u^{p+1} \mathrm{~d} r\right)^{2} \leq \lambda(N+2) \int_{0}^{1} r^{N-1} u(r) \mathrm{d} r=o(\lambda) .
$$


Thus we obtain

which proves the lemma.

$$
\int_{0}^{1} r^{N-1} u^{p+1} \mathrm{~d} r \leq o(\sqrt{\lambda})
$$

LemMA 11. If $u(r, \lambda, d)$ is a positive solution to (1.2), (1.3) and (1.5) then for $\lambda \in[0,1]$ we have

$$
\left\|u_{\lambda}(r, \lambda, d)+\frac{r^{2}}{2 N}\right\|_{\infty} \leq o\left(\lambda^{p /(2(p+1))}\right)
$$

and, hence,

$$
\lim _{d \rightarrow+\infty}\left\|u_{\lambda}(r, \lambda, d)+\frac{r^{2}}{2 N}\right\|_{\infty}=0
$$

Proof. From the definition of $u_{\lambda}$ we have

$$
\begin{aligned}
u_{\lambda}(r, \lambda, d) & =-\int_{0}^{r} s^{-N+1} \int_{0}^{s} r^{N-1}\left(1+(p+1)|u|^{p} u_{\lambda}\right) \mathrm{d} r \mathrm{~d} s \\
& =-\frac{r^{2}}{2 N}-\int_{0}^{r} s^{-N+1} \int_{0}^{s} r^{N-1}(p+1)|u|^{p} u_{\lambda} \mathrm{d} r \mathrm{~d} s .
\end{aligned}
$$

Since $-u_{\lambda} \leq 1 / 2 N$ (see lemma 7), from (2.30) we obtain that

$$
u_{\lambda}(t, \lambda, d) \leq-\frac{r^{2}}{2 N}+\frac{p+1}{2 N} \int_{0}^{1} s^{-N+1} \int_{0}^{s} r^{N-1} u^{p} \mathrm{~d} r \mathrm{~d} s .
$$

Hence, it suffices to show that $\int_{0}^{s} r^{N-1} u^{p} \mathrm{~d} r \rightarrow 0$ as $d \rightarrow \infty$. Now by Hölders inequality we have

$$
\int_{0}^{s} r^{N-1} u^{p} \mathrm{~d} r \leq \int_{0}^{1} r^{N-1} u^{p} \mathrm{~d} r \leq M\left(\int_{0}^{1} r^{N-1} u^{p+1}\right)^{p /(p+1)} .
$$

Now using lemma 10 we obtain that

$$
\int_{0}^{1} r^{N-1} u^{p} \mathrm{~d} r \leq o\left(\lambda^{p /(2(p+1))}\right)
$$

Therefore,

$$
\int_{0}^{r} s^{-N+1} \int_{0}^{s} r^{N-1}(p+1) u^{p}\left(-u_{\lambda}\right) \leq\left(\frac{1+p}{2 N}\right) \int_{0}^{r} s^{-N+1} \int_{0}^{s} r^{N-1} u^{p} \mathrm{~d} r=o\left(\lambda^{p /(2(p+1))}\right)
$$

and, hence, $u_{\lambda}(r) \leq-r^{2} / 2 N+o\left(\lambda^{p /(2(p+1))}\right)$ which, in turn, implies

$$
\left\|u_{\lambda}(r, \lambda, d)+\frac{r^{2}}{2 N}\right\|_{\infty} \leq o\left(\lambda^{p /(2(p+1))}\right),
$$

thus, completing the proof. 
Now we show that $u_{d}(1, \lambda, d) \neq 0$. Consider the initial value problem (1.2), (1.3) and (1.5) for $\lambda=0$. That is

$$
\begin{gathered}
v^{\prime \prime}+\frac{N-1}{r} v^{\prime}+|v|^{p} v=0, \\
v(0)=d \quad \text { and } \quad v^{\prime}(0)=0 .
\end{gathered}
$$

That is we denote $u(r, d, 0)$ by $v(r, d)$. From Pohozaev's identity $(2.5)$ and the quadratic formula we obtain

$$
v^{\prime}=-\frac{N-2}{2 r} v-\frac{N-2}{2 r} v \sqrt{1-\frac{4 r^{2}}{N(N-2)} v^{p}}
$$

for all $r>\hat{r}=K_{1} d^{-p / 2}$, where $K_{1}$ is a constant independent of $d$ (see lemma 5). Also, given an $\varepsilon>0$, there exists a constant $K_{2}$ independent of $d$ such that for $r \geq k_{2} d^{-p / 2}$ we have

$$
\frac{2}{N(N-2)} r^{2} v^{p} \leq \varepsilon
$$

Substituting (2.34) in (2.33) we get

$$
v^{\prime}(r) \leq-\sigma \frac{v}{r},
$$

where $\sigma=\frac{1}{2}(N-2)(1+\sqrt{1-\varepsilon})$. Integrating this over $[r, 1]$ we obtain

From this we infer

$$
v(r) \geq v(1) r^{-\sigma} \text {. }
$$

$$
\int_{k d^{-p / 2}}^{1} r^{N-1} v(r) \mathrm{d} r \geq(N-\sigma)^{-1} v(1)\left[1-\left(k d^{-p / 2}\right)^{N-\sigma}\right] .
$$

Thus, by choosing $\varepsilon$ small enough and $d$ large enough we get

$$
\int_{0}^{1} r^{N-1} v(r) \mathrm{d} r \geq v(1) \tau,
$$

with $\tau<\frac{1}{2}$ but arbitrarily close to $\frac{1}{2}$. Suppose now that $u_{d}(1, \lambda, d)=0$. Then using the mean value theorem we obtain

$$
v(1)=u(1,0, d)=u(1, \lambda, d)-\lambda u_{\lambda}(1, \hat{\lambda}, d)
$$

for some $\hat{\lambda} \in[0, \lambda]$. From lemma 11 and the fact that $u(1, \lambda, d)=0$, we obtain

$$
v(1)=\lambda\left(\frac{1}{2 N}-o\left(\lambda^{p /(2(1+p))}\right)\right)
$$

From lemma 11 and the rescaling equation in (2.18), we obtain

$$
u^{\prime}(1, d, \lambda)=\lambda\left(\frac{N+2)}{2}\right)\left(-\frac{1}{2 N}+o\left(\lambda^{p /(2(1+p))}\right)\right) .
$$


From Pohozaev's identity and (2.5) we have

$$
u^{\prime}(1, d, \lambda)=-\sqrt{\lambda(N+2) \int_{0}^{1} r^{N-1} u(r) \mathrm{d} r}
$$

and by the mean value theorem and lemma 3 we get

$$
u(r, \lambda, d) \geq v(r, d)-\frac{\lambda}{2 N} r^{2}
$$

Combining (2.40) and (2.41) we obtain

$$
\lambda(N+2) \int_{0}^{1} r^{N-1} u(r) \mathrm{d} r=\left(\frac{N+2}{2} \lambda\right)^{2}\left(\frac{1}{4 N^{2}}+o\left(\lambda^{p /(2(1+p))}\right)\right) .
$$

That is,

$$
\int_{0}^{1} r^{N-1} u(r) \mathrm{d} r=\left(\frac{N+2}{4} \lambda\right)\left(\frac{1}{4 N^{2}}+o\left(\lambda^{p /(2(1+p))}\right)\right) .
$$

On the other hand, from (2.38), (2.39) and (2.42) we obtain

$$
\begin{aligned}
\int_{0}^{1} r^{N-1} u(r) \mathrm{d} r & \geq \int_{0}^{1} r^{N-1}\left(v(r)-\frac{\lambda}{2 N} r^{2}\right) \mathrm{d} r>v(1) \tau-\frac{\lambda}{2 N(N+2)} \\
& =\lambda\left[\frac{1}{2 N}-o\left(\lambda^{p /(2(1+p))}\right)\right] \tau-\frac{\lambda}{2 N(N+2)} .
\end{aligned}
$$

Combining (2.43) and (2.44) we obtain

$$
o\left(\lambda^{p /(2(1+p))}\right) \geq \frac{(8 \tau-1) N^{2}+(16 \tau-12) N-4}{16 N^{2}(N+2)} .
$$

Since $\tau$ can be chosen arbitrarily close to $\frac{1}{2}$, we see that the numerator of the right-hand side can be made arbitrarily close to $3 N^{2}-4 N-4$, which is positive for $N \geq 3$. Hence, (2.45) cannot hold for small values of $\lambda$ which is a contradiction. Thus, there exist $D>0$ and $\Lambda>0$ such that if $u(\cdot, \lambda, d)$ is a positive solution to (1.2), (1.3), (1.5), $\lambda \in(0, \Lambda)$ and $d>D$ then

$$
u_{d}(1, \lambda, d)<0 \text {. }
$$

\section{PROOF OF THEOREM 1}

Since $u_{\lambda}(1, \lambda, d)<0$ (see lemma 7 ), the implicit function theorem implies that if $S$ is a connected component of $\{(\lambda, d) ; u(1, \lambda, d)=0, u(r, \lambda, d)>0$ for all $r \in[0,1]\}$ then there exists a differentiable function $F:(0, \infty) \rightarrow(0, \infty)$ such that $S=\{(F(d), d) ; d \in(0, \infty)\}$. Integrating (1.2) on $[0,1]$ we see that $-d \leq-F(d) / 2 N$. Hence,

$$
\lim _{d \rightarrow 0} F(d)=0
$$

Let us see now that

$$
\lim _{d \rightarrow \infty} F(d)=0
$$


By lemma 9 we have $\lim _{d \rightarrow \infty} u(1 / 4, F(d), d)=0$. Thus, if $\lim \sup _{d \rightarrow \infty} F(d)>0$ then for some sequence $\left\{d_{n}\right\} \rightarrow \infty$ we have $\left\{F\left(d_{n}\right) / u(1 / 4, F(d), d)\right\} \rightarrow \infty$. Hence, because $u\left(\cdot, F\left(d_{n}\right), d_{n}\right)$ satisfies

$$
u^{\prime \prime}+\frac{N-1}{r} u^{\prime}+\left(|u|^{p}+\frac{F\left(d_{n}\right)}{u}\right) u=0,
$$

by the Sturm comparison theorem we see that $u\left(\cdot, F\left(d_{n}\right), d_{n}\right)$ must have a zero in $[1 / 4,1)$. This contradicts that $u\left(\cdot, F\left(d_{n}\right), d_{n}\right)$ is positive in $(0,1)$. Thus, (3.2) is proven.

Since $u(r, F(d), d) \leq d$ for all $r \in[0,1]$, by the Sturm comparison theorem we see that for $d>0$ small $u_{d}(r, F(d), d)>0$ for all $r \in[0,1]$. hence, by the implicit function theorem there exists $\delta>0$ and an increasing differentiable function $\phi:(0, \delta) \rightarrow(0, \infty)$ such that $u(\cdot, \lambda, d)$ is a solution to (1.2), (1.3), (1.5) if and only if $d=\phi(\lambda)$. Thus, if $S_{1}=\{(F(d), d) ; d \in(0, \infty)\}$ and $S_{2}=\left\{\left(F_{1}(d), d\right) ; d \in(0, \infty)\right\}$ are connected components of positive solutions to (1.2), (1.3), (1.5), by (3.1) we see that $d=\phi(F(d))=\phi\left(F_{1}(d)\right)$. Hence, $F(d)=F_{1}(d)$ for $d$ close to 0 . Therefore, $S_{1}=S_{2}$, which proves that the set of positive solutions to (1.1) is connected.

Differentiating $u(1, \lambda(d), d))=0$ with respect to $d$ we obtain

$$
\left.\left.u_{d}(1, \lambda(d), d)\right)+u_{\lambda}(1, \lambda(d), d)\right) \cdot \lambda^{\prime}(d)=0 \text {. }
$$

This, (2.46) and (3.2) imply that $F$ is a decreasing function in $(D, \infty)$, which proves that for $\lambda<F(D)$ the problem $(1.1)$ has exactly one solution with $u(0)>D$. Since $\phi$ is an increasing function, so is $F$. Thus, if $\lambda \in(0, \phi(\delta))$ then (1.1) has exactly one small solution. Thus, if $0<\lambda<\min \{\delta / 2, F(D), \min \{F(d) ; d \in[\delta / 2, F(D]\}\}$ then the problem (1.1) has exactly two positive solutions.

\section{REFERENCES}

1. ADAMS R., Sobolev Spaces. Academic Press, New York (1975).

2. POHOZAEV S. I., Eigenfunctions of the equation $\Delta u+\lambda f(u)=0$, Soviet Math. Dokl. 6, 1408-1411 (1965).

3. ATKINSON F., BREZIS H. \& PELETIER L., Solutions d'equations elliptiques avec exposant critique qui changent de signe, C. r. Acad. Sci. Paris, Serie I 306, 711-714 (1988).

4. BREZIS H. \& NIRENBERG L., Positive solutions of nonlinear elliptic equations involving critical Sobolev exponents, Communs pure appl. Math. 36, 437-447 (1983).

5. CASTRO A. \& KUREPA A., Radially symmetric solutions to a Dirichlet problem involving critical exponents, Trans. Am. math. Soc. (to appear).

6. CERAMI G., SOLIMINI S. \& STRUWE M., Some existence results for superlinear elliptic boundary value problems involving critical exponents, $J$. funct. Analysis 8, 289-306 (1986).

7. GIDAS B., NI W. M. \& NIRENBERG L., Symmetry and related properties via the maximum principle, Communs math. Phys. 68, 209-243 (1979).

8. TARANTELLO G., On nonhomogeneous elliptic equations involving critical Sobolev exponent, $A n n$. Inst. $H$. Poincaré Analyse non Lineaire 9, 281-304 (1992).

9. PUCCI P. \& SERRIN J., A general variational identity, Indiana Univ. Math. J. 35(3), 681-703 (1986).

10. INCE E. L., Ordinary differential equations. Dover, New York (1956). 